\title{
Ovine Sinus Tumor
}

National Cancer Institute

\section{Source}

National Cancer Institute. Ovine Sinus Tumor. NCI Thesaurus. Code C134781.

A neoplasm that occurs in the sinus of a sheep. 